\title{
Overhauser-Effekt und Relaxation in organischen Festkörperradikalen
}

\author{
J. Haupt * und W. Müller-Warmuth * \\ Max-Planck-Institut für Chemie (Otto-Hahn-Institut), Mainz \\ (Z. Naturforschg. 21 a, 158-164 [1966] ; eingegangen am 20. September 1965) \\ Herrn Professor J. Маттаuch zum 70. Geburtstag gewidmet
}

\begin{abstract}
The dynamic proton polarization, and the proton and electron spin relaxations have been studied in the organic polycrystalline free radicals bis-diphenylene-p-chlor-phenyl allyl (BPA), diphenyl picryl hydrazyl (DPPH), Wurster's blue perchlorate (WBP) and galvinoxyl (GAV). Enhancement factors between +370 and +79 were observed; extrapolation to infinite microwave power yielded values near the theoretical $\gamma_{\mathrm{S}} / \gamma_{\mathrm{I}}$ ratio. Electron spin-lattice relaxation times are of the order of $10^{-7}$ to $10^{-8} \mathrm{sec}$; proton relaxation times are of the order of $10^{-3} \mathrm{sec}$. With the exception of galvinoxyl at low temperatures, the proton resonance line shapes are not much influenced by the local field of the unpaired electrons. The ESR spectra show a typical exchange-narrowing. All the results are discussed in terms of a scalar nuclear-electron interaction in which electron-electron exchange couplings provide a time dependence of random character.
\end{abstract}

Obwohl der erste experimentelle Nachweis einer dynamischen Kernpolarisation durch OverhauserEffekt in einem Nichtmetall an dem organischen freien Radikal DPPH gelang ${ }^{2}$, enthält die Literatur widerspruchsvolle Ergebnisse über Art und Stärke der erreichbaren Protonenpolarisation ${ }^{2-7}$. Der Grund liegt darin, daß bei den zur Messung der Protonenresonanz benutzten kontinuierlichen Einstrahlmethoden Linienverformungen und Seitenresonanzen entstanden, sobald die Mikrowellenleistung zur Sättigung der Elektronenresonanz eine gewisse Stärke erreichte. Die beobachteten Effekte wurden von Winter als Mehrquantenübergänge zwischen den beiden Elektronenresonanzniveaus des DPPH erklärt ${ }^{8}$. Dadurch werden Absorptionen und Emissionen vorgetäuscht, die nichts mit der KernElektron-Kopplung zu tun haben, und die sich dem vergrößerten Kernresonanzsignal überlagern. Eine zweite experimentelle Schwierigkeit, die für die relativ niedrigen Vergrößerungsfaktoren - etwa 20 bei Beljers et al. ${ }^{2}$, 6 bei Uebersfeld et al. ${ }^{3}, 4$ bei Berthet ${ }^{4}$ und 16 bei Tschao ${ }^{5,6}$ - verantwortlich sein dürfte, ist die starke Dämpfung, die die Resonanzabsorption im Mikrowellenresonator erzeugt. Trotz Anwendung stärkerer Mikrowellenleistungen wachsen dadurch die hochfrequenten Sättigungsfelder nicht genügend an, um starke Protonenpolarisationen zu erzeugen.

Andererseits hat $\mathrm{W}_{\mathrm{EBB}}$ gezeigt, daß in Festkörperradikalen durchaus Vergrößerungsfaktoren

* Gegenwärtige Adresse: EURATOM CCR, Ispra (Italien).

1 A. W. Overhauser, Phys. Rev. 92, 411 [1953].

2 H. G. Beljers, L. van der Kint u. J. S. v. Wieringen, Phys. Rev. 95, 1683 [1954].

3 E. Erb, J. L. Motchane u. J. Uebersfeld, C. R. Acad. Sci. Paris 246, 3050 [1958].

${ }^{4}$ G. Berthet u. R. Gendrin, Arch. Sci. Genève 11, 272 [1958]. von etwa 55 (bei Wursters Blau) oder knapp 50 bei 1,3-Bis-diphenylen-2-phenylallyl) möglich sind ${ }^{9}$. Die Kenntnis der charakteristischen Eigenschaften der dynamischen Kernpolarisation ist nötig, um die Kern-Elektron-Kopplung, die elektronischen Austauschprozesse, und um die Kernrelaxation in paramagnetischen Substanzen zu verstehen. Außerdem werden die freien Radikale im Rahmen einer umfassenderen Untersuchung der dynamischen Polarisation in Festkörpern zum Einbau in andere organische Materialien benutzt.

Aus diesen Gründen wurde in der vorliegenden Arbeit die dynamische Protonenpolarisation in den polykristallinien organischen freien Radikalen Bisdiphenylen-p-chlor-phenylallyl (BPA), Diphenylpikrylhydrazyl (kristallflüssigkeitsfrei) (DPPH), Wursters Blau-Perchlorat (WBP) und Galvinoxyl (GAV) studiert. Zur Vermeidung der oben erwähnten Schwierigkeiten wurde zum Nachweis der Protonenresonanz eine Impulsmethode benutzt, bei der das Signal in Abwesenheit eines bei der Protonenresonanz eingestrahlten Hochfrequenzfeldes gemessen wird. Außerdem wurden sehr kleine Substanzmengen in den Resonator gegeben, um die Begrenzung der Mikrowellenfeldstärke durch Resonatorverluste wie Wandströme und dielektrische Bedämpfung und nicht durch die paramagnetische Resonanzabsorption zu bedingen. Nur auf diese Weise ist es möglich, die dynamische Polarisation quantitativ in Abhängigkeit von der Mikrowellenfeldstärke zu untersuchen.

\footnotetext{
5 Y. H. Tschao u. J. Hervé, C. R. Acad. Sci. Paris 250, 700 [1960].

${ }_{6}^{6}$ Y. H. Tschao, C. R. Acad. Sci. Paris 252, 1765 [1961].

7 A. V. Kessenikh, Soviet Phys.-JETP 13, 21 [1961].

8 J. M. Winter, J. Phys. Radium 19, 802 [1958].

9 R. H. WEBB, Phys. Rev. Letters 6, 611 [1961].
} 


\section{Experimentelle Methodik}

Ein Blockschaltbild der Apparatur zeigt Abb. 1. Einige Milligramm der Radikalprobe befinden sich in einem $H_{102}$-Mikrowellenresonator, der auf einer Frequenz von etwa $9,6 \mathrm{GHz}$ schwingt. Die Leistung wird in einem $\frac{1}{2} \mathrm{~W}$ - (V a ri a n VA 242) bzw. 4 W-Klystron

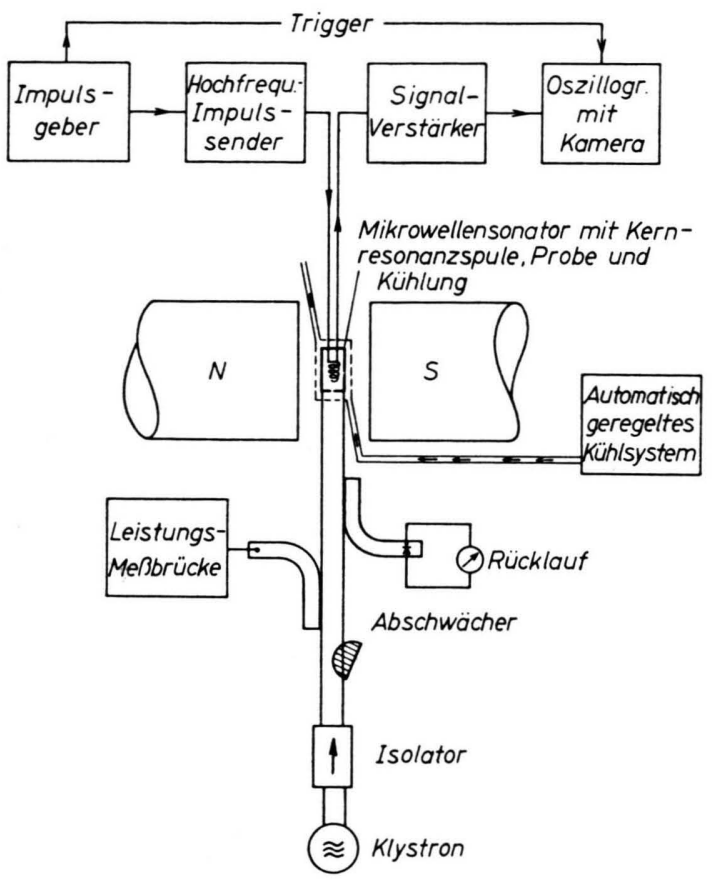

Abb. 1. Blockschaltbild der Apparatur; obere Hälfte: Kern. resonanz-Impulsspektrometer zum Nachweis der Protonen resonanz; unten: Mikrowelleneinheit zur Sättigung der Elektronenresonanz.

(V a r i a n V 23) erzeugt und über einen Isolator direkt auf den über eine Irisblende angekoppelten Resonator gegeben. Zum Studium der Leistungsabhängigkeit der dynamischen Kernpolarisation ist ein variables Dämpfungsglied vorgesehen; die Mikrowellenleistung wird im Nebenschluß gemessen.

Zur Messung der durch dynamische Polarisation vergrößerten und der unvergrößerten Protonenresonanzsignale dient ein einfaches Festkörper-Impulsspektrometer, dessen wesentliche Eigenschaften bereits früher beschrieben wurden ${ }^{10}$. Es wird hier zum Studium der Spin-Gitter-Relaxationszeiten $T_{1}$ und zur Bestimmung der relativen Signalintensitäten benutzt. Das letztere geschieht durch Beobachtung des freien Präzessionssignals, das einem kurzen kräftigen $90^{\circ}$-Impuls folgt. Da sich die Form der Protonenresonanz und damit die Form der abklingenden Transversalmagnetisierung durch Erzeugung einer dynamischen Polarisation nicht mit der Sättigungsleistung ändert, kann der Vergröße-

10 J. Haupt, W. Müller-W $\mathbb{W}_{\text {armuth }}$ u. G. Schulz, Z. Angew. Phys. 18, 132 [1964]. rungsfaktor in gewissen Grenzen direkt aus dem $\mathrm{Si}$ gnalvergleich bestimmt werden. Wichtigste Voraussetzung dafür ist die Linearität des Empfängers. Auf diese wurde beim Aufbau des Signalverstärkers Wert gelegt. Durch Aufnahme der Verstärkercharakteristik wurde der lineare Bereich der Verstärkung ermittelt. Damit nicht Eingangssignale sehr verschiedener Intensität in der Elektronik des Spektrometers verarbeitet werden müssen, was zweifellos zu einer unzulässigen Überschreitung des Linearitätsbereichs führen würde, wird bei den durch dynamische Polarisation vergrößerten Signalen eine definierte Abschwächung nach der ersten und zweiten Verstärkerstufe vorgenommen. Das geschieht durch geeichte Hochfrequenzspannungsteiler. Die auf dem Oszillographenschirm photographisch ausgewertete Ausgangsspannung bleibt dadurch immer etwa gleich.

Das wesentliche experimentelle Problem ist die gleichzeitige Heranführung von zwei magnetischen Hochfrequenzfeldern an die Substanzprobe: das eine bei $9,6 \mathrm{GHz}$ zur Anregung der Elektronenresonanz, das andere bei etwa $14,5 \mathrm{MHz}$ in Form von Impulsen, welche die Kernmagnetisierung in die Beobachtungsebene kippen. Die beiden Felder sind senkrecht zum statischen Magnetfeld (etwa 3500 Gauss) gerichtet und sollen sich gegenseitig möglichst wenig stören. Der Energieverlust im Resonator soll klein gehalten, und die Füllfaktoren groß gemacht werden. Diese Forderungen können nur in Form eines Kompromisses erfüllt werden. Die hier gewählte Lösung zeigt Abb. 2.

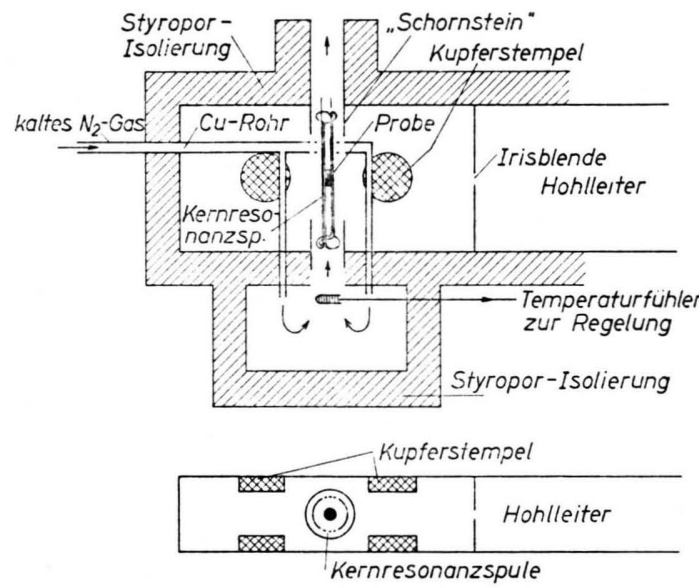

Abb. 2. Schema des Mikrowellenresonators mit Kernresonanzspule und Kühlgas-Durchflußsystem im Schnitt und Aufblick. Zur Veranschaulichung der Windungsanordnung ist die Spule perspektivisch gezeichnet.

Durch Anbringen von zwei Kupferstempeln beiderseits des Probenortes wurde der Mikrowellenresonator kapazitiv belastet. Dadurch erreicht man bei konstanter Eigenfrequenz eine Verkleinerung des Resonators, und damit bei gleicher Eingangsleistung eine höhere Mikrowellenfeldstärke. Die magnetischen Feldlinien werden 
durch die gewählte Geometrie auf den Probenbereich konzentriert, die elektrischen etwas herausgezogen. Die Kernresonanz-Nachweisspule ist so gewickelt, daß keine $H_{1 \mathrm{e}}$-Mikrowellen-Feldlinien hindurchgreifen. „Kupferschornsteine" um die Probenführung verhindern, daß dies an den Windungsenden geschieht, an denen die Verbindungen der einzelnen Windungen senkrecht zur Feldrichtung liegen. Auf diese Weise konnte eine völlige Entkopplung der Spule - allerdings verbunden mit dem Nachteil eines nicht sehr guten Füllfaktors für die Kernresonanz - erreicht werden.

Zur Einstellung der Probentemperatur und zur Abführung der durch die Mikrowellenleistung erzeugten Wärme dient ein Temperaturregelsystem. Es erlaubt die Einstellung von Temperaturen zwischen $77^{\circ} \mathrm{K}$ und Zimmertemperatur. Das Prinzip, der Wärmeaustausch und die Regelung sind an anderer Stelle erklärt ${ }^{10}$. Ein Unterschied besteht im Durchflußsystem des Kühlgases. Dieses $\left(\mathrm{N}_{2}\right)$ strömt hier zunächst durch Kupferrohre, die fest mit dem Resonator verlötet sind und ihn mit abkühlen. Der in Abb. 2 eingezeichnete Kühlgasbehälter aus Stryropor hat sich zur Abscheidung von flüssigen Anteilen bei Temperaturen in der Nähe von flüssigem $\mathrm{N}_{2}$ als nützlich erwiesen. Unmittelbar an der Probe selbst streicht dann nur noch Gas vorbei, das keine unregelmäßigen Störungen durch verschieden starke Resonatordämpfung oder -verstimmung erzeugt. Der Temperaturfühler zur Regelung befindet sich am oberen Ende des Behälters, die genaue Probentemperatur bei einer bestimmten Einstellung des Reglers wird durch Blindproben ermittelt. Sie unterscheidet sich nur wenig von der Temperatur des Fühlers für die Regelung.

Ein wichtiges Problem ist die vollständige Abführung der ganzen in der Probe entwickelten Wärme. In den untersuchten Radikalen, besonders bei DPPH, fand wegen der starken elektronischen Spin-Gitter-Wechselwirkungen eine kräftige magnetische Aufheizung der Probe unter Resonanzbedingung statt. Bei den Experimenten wurde deshalb die Resonanzbedingung durch Variation des Magnetfeldes überhaupt nur in Zeiträumen hergestellt, die klein gegenüber $1 \mathrm{~s}$ waren. Der Kernresonanznachweis erfolgte dabei etwa alle $10 \mathrm{~ms}$.

Aus den in der Einführung erwähnten Gründen konnten nur so kleine Substanzmengen verwendet werden, daß eine direkte Bestimmung des unvergrößerten Protonenresonanzsignals nicht mehr möglich war. Die Gleichgewichtspolarisation mußte daher an einer größeren Radikalprobe und aus dem bekannten Gewichtsverhältnis ermittelt werden. Kernresonanz-Kontrollmessungen an verschieden großen Proben haben die Zulässigkeit dieses Verfahrens sichergestellt.

Die in Abb. 2 angegebene Doppelresonanzanordnung wurde eigentlich für Untersuchungen größerer Probenvolumina entwickelt und könnte aus diesem Grunde noch etwas abgewandelt werden, wenn man sie nur für die vorliegenden Studien benutzen wollte.

11 A. Abragam, J. Combrisson u. I. Solomon, C. R. Acad. Sci. Paris 245, 157 [1957].

\section{Meßergebnisse}

Die Frequenzabhängigkeit des Vergrößerungsfaktors der dynamischen Protonenpolarisation in den untersuchten Radikalen zeigt Abb. 3 für eine bestimmte konstante Mikrowellenleistung. Zur Messung dieser Kurven wurde die Elektronenresonanzfrequenz konstant gehalten und das Magnetfeld zusammen

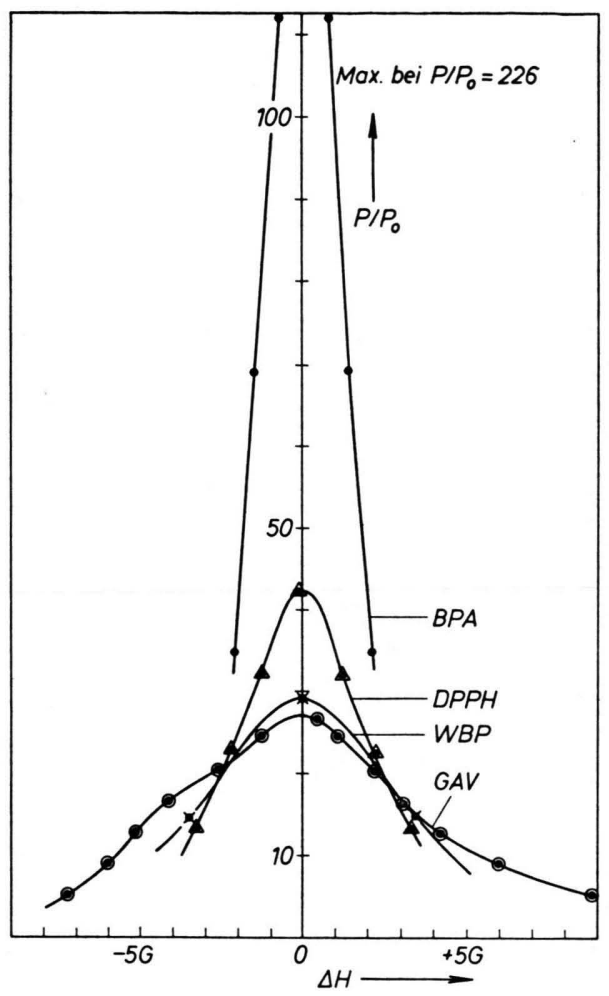

Abb. 3. Meßpunkte für den Vergrößerungsfaktor der dynamischen Protonenpolarisation bei einer Mikrowellenleistung von $660 \mathrm{~mW}$.

mit der Protonenfrequenz um den Mittelwert der maximalen Polarisation herum stufenweise geändert. Die Polarisation ist immer positiv. Bei schwacher Mikrowelleneinstrahlung entsprechen die Linienformen der gewöhnlichen Elektronenresonanz. Lediglich bei Galvinoxyl deuten sich im Abstand von etwa 4. Gauss geringfügige Ausbuchtungen an. Davon abgesehen zeigen die Kurven, daß es sich bei der dynamischen Kernpolarisation in allen Fällen um einen reinen Overhauser-Effekt handelt.

$\mathrm{Zu}$ dessen Beschreibung soll wie in Flüssig. keiten ${ }^{11,12}$ die Beziehung

12 J. Haupt u. W. Müller-W 1011 [1962]. 


$$
P / P_{0}=1-\varrho f s \gamma_{S} / \gamma_{1}=1-F s
$$

benutzt werden. Der Kern-Elektronen-Kopplungsparameter muß nach Abb. 3 negativ sein. Mit den gyromagnetischen Verhältnissen für (freie) Elektronen und Protonen ist $\gamma_{S} / \gamma_{I}=658$. Der Streufaktor $f$ ist kleiner als eins, wenn die Relaxation der Protonen nicht ausschließlich über die Wechselwirkung mit den Elektronen erfolgt. Zur Ermittlung des "Sättigungsfaktors" $s$ wurde die Abhängigkeit des Vergrößerungsfaktors $P / P_{0}$ von der Mikrowellenleistung gemessen. Abb. 4 zeigt das Ergebnis für zwei Beispiele. Darin ist $1 /\left(P / P_{0}-1\right)$ gegen die inverse Mikrowellenleistung aufgetragen, weil man

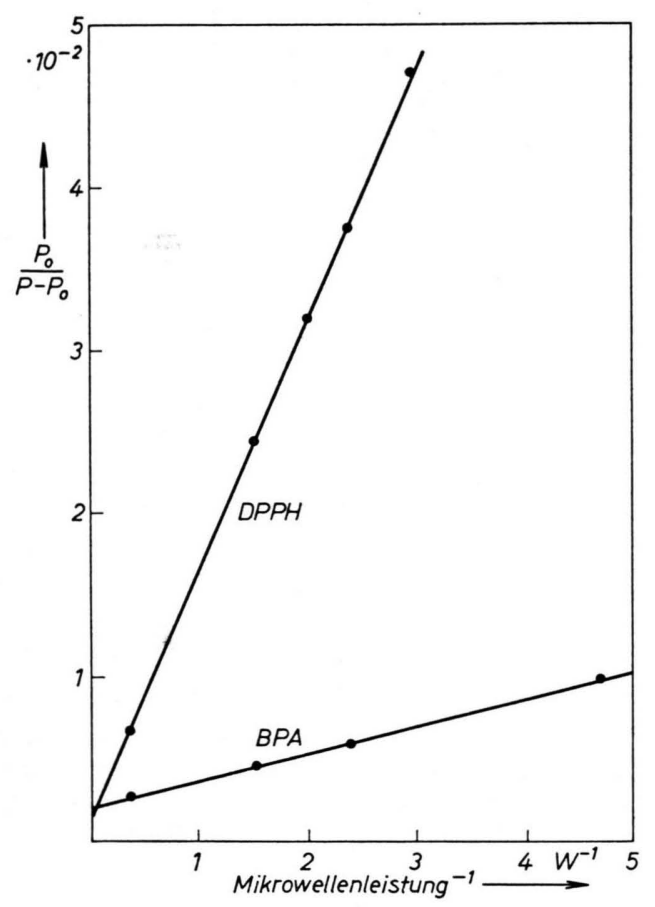

Abb. 4. Beispiele von „Sättigungskurven“. Die Meßwerte des Vergrößerungsfaktors der dynamischen Protonenpolarisation bei verschieden starker Anregung sind in der Form $1 /\left(P / P_{0}-1\right)$ gegen (Mikrowellenleistung) ${ }^{-1}$ aufgetragen. Der Schnitt der durch die Meßpunkte gelegten Geraden mit der Ordinate liefert den extrapolierten Vergrößerungsfaktor.

in dieser Darstellung vielfach eine Gerade erhält ${ }^{12}$, deren Schnittpunkt mit der Ordinate den (auf unendliche Mikrowellenleistung) extrapolierten Vergrößerungsfaktor $(s=1)$ liefert. Die Ergebnisse für die extrapolierten Vergrößerungsfaktoren $F$ sind zusammen mit den tatsächlich gemessenen und anderen Daten in Tab. 1 zusammengestellt. Die Genauigkeit dieser Extrapolation ist allerdings wesentlich schlech-

\begin{tabular}{|c|c|c|c|c|}
\hline Radikal & $\begin{array}{c}\left(P / P_{0}\right)_{\max } \\
\text { gemessen }\end{array}$ & $-F$ & $T_{1 \mathrm{e}} / \mathrm{sec}$ & $T_{2 \mathrm{e}}^{*} / \mathrm{sec}$ \\
\hline BPA & +370 & $505 \pm 50$ & $7,8 \cdot 10^{-8}$ & $7,8 \cdot 10^{-8}$ \\
DPPH & +149 & $650+80$ & $3,2 \cdot 10^{-8}$ & $2,5 \cdot 10^{-8}$ \\
WBP & +147 & $800 \pm 200$ & $3,3 \cdot 10^{-8}$ & $1,7 \cdot 10^{-8}$ \\
GAV & +79 & $400 \pm 100$ & $6 \cdot 10^{-8}$ & $1,3 \cdot 10^{-8}$ \\
\hline
\end{tabular}

Tab. 1. Meßergebnisse der dynamischen Protonenpolarisation und der elektronischen Relaxation für $t=24^{\circ} \mathrm{C}, \omega_{S}=6,2 \cdot 10^{10}$ $\mathrm{s}^{-1}, \omega_{I}=0,94 \cdot 10^{8} \mathrm{~s}^{-1}$.

ter als bei früheren Untersuchungen in Lösungen. Das liegt daran, daß die Stärke der Mikrowelleneinstrahlung wegen zu großer und durch das Temperaturregelsystem nicht mehr wegführbarer Wärmeentwicklung begrenzt werden mußte. Bei WBP ergab sich zudem in der der Abb. 4 analogen Darstellung keine Gerade.

Aus den „Sättigungskurven“ erhält man das Produkt der elektronischen Relaxationszeiten. Wie früher gezeigt wurde ${ }^{12}$, gilt solange $\gamma_{\mathrm{s}}^{2} H_{1 \mathrm{e}}^{2} T_{1 \mathrm{e}} T_{2 \mathrm{e}} \ll 1$ (bzw. $\left.\left|P / P_{0}-1\right| \ll|F|\right)$ ist:

$$
T_{1 \mathrm{e}} T_{2 \mathrm{e}}^{*}=\frac{P / P_{0}-1}{-F} \frac{1}{\gamma_{S^{2}} H_{1 \mathrm{e}}^{2}} .
$$

Bei ausschließlich homogen verbreiterten ESR-Linien steht für $T_{.2 \mathrm{e}}^{*}$ die transversale Relaxationszeit $T_{2 \mathrm{e}}$. (2) ist eigentlich für Lösungen mit austauschverschmälerter Hyperfeinstruktur hergeleitet worden, gilt aber sinngemäß auch für die hier studierten Festkörperradikale. Deren Linien sind ebenfalls stark austauschverschmälert und besitzen höchstens eine inhomogene Verbreiterung, die die Kurvenform nicht stark verändert, und die sich durch eine effektive Relaxationszeit $T_{2 \mathrm{e}}^{*}$ beschreiben läßt. Zur Bestimmung des Mikrowellenmagnetfeldes $H_{1 \mathrm{e}}$ in $\mathrm{Ab}$ hängigkeit von der Leistung wurde für BPA in Übereinstimmung mit der Sättigungsverbreiterung der LoRENTz-förmigen ESR-Linie $T_{1 \mathrm{e}}=T_{2 \mathrm{e}}$ angenommen und $T_{2 \mathrm{e}}$ aus der ESR-Linienbreite ermittelt. $\mathrm{Da} \beta$ diese Annahme nicht sehr falsch ist, wird durch das mit dieser Eichung erhaltene $T_{1 \mathrm{e}}$ für DPPH bestätigt. Ein zu groß vorgetäuschtes $H_{1 \mathrm{e}}$ würde zu kurze $T_{1 \mathrm{e}}$ liefern, die dann fälschlicherweise kürzer als $T_{2 \mathrm{e}}$ werden können. Die Ergebnisse für $T_{1 \mathrm{e}}$ und $T_{2 \mathrm{e}}$ sind ebenfalls in Tab. 1 enthalten.

Alle diese Studien erfolgten bei Zimmertemperatur. Wegen der besonderen Schwierigkeiten durch die Aufheizung der Probe wurde das Verhalten bei tieferen Temperaturen nur qualitativ untersucht. Für den Overhauser-Effekt besteht in allen Fällen bis zur Temperatur des flüssigen Stickstoffs keine sehr starke Temperaturabhängigkeit. 
Da es sich bei der dynamischen Kernpolarisation um einen Overhauser-Effekt handelt, erwartet man unter Umständen aus Daten über die Spin-GitterRelaxationszeit $T_{1}$ der Protonen in den Radikalen

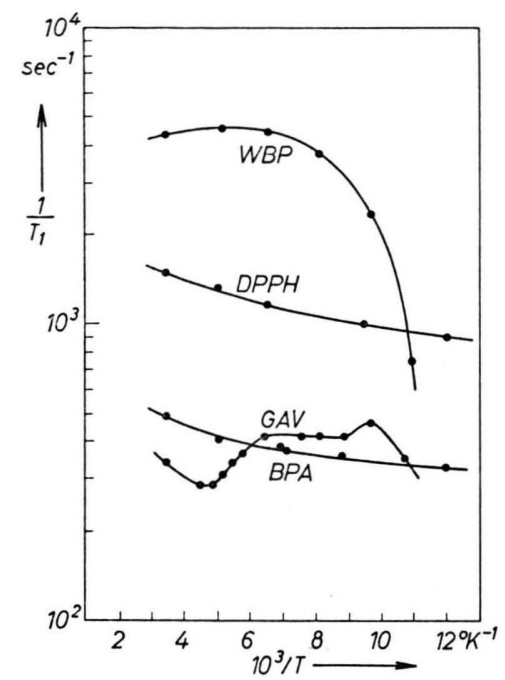

Abb. 5. Temperaturabhängigkeit der Protonen-Spin-GitterRelaxationsraten.

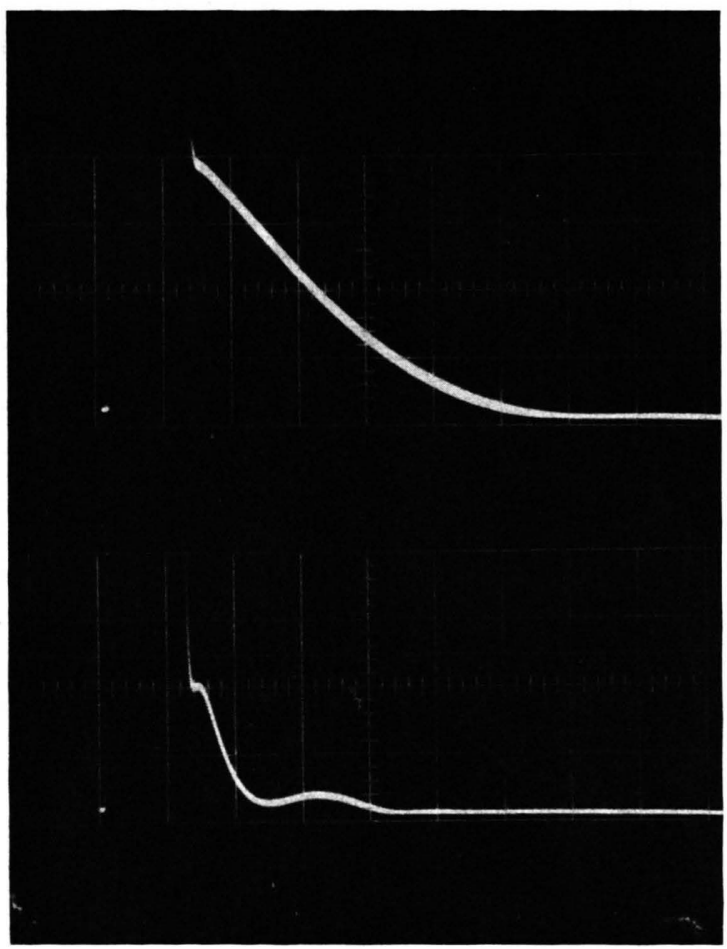

Abb. 6. Linienform der Protonenresonanzsignale nach einem $90^{\circ}$-Impuls in Galvinoxyl; oben: bei $-50^{\circ} \mathrm{C}$, unten: bei $-180^{\circ} \mathrm{C}$. In der Zeitachse entspricht eine Einheit des Strichnetzes $10 \mu \mathrm{s}$. ergänzende Informationen über den Wechselwirkungsmechanismus. Meßergebnisse für die Temperaturabhängigkeit der Relaxationsraten gibt Abb. 5 .

Die Linienform der Protonenresonanzen ist in allen Fällen ähnlich, für den zeitlichen Abfall nach einem $90^{\circ}$-Impuls zeigt Abb. 6 , oben ein Beispiel. Auch besteht mit der Ausnahme von GAV keine Temperaturabhängigkeit der Linienform. Bei Galvinoxyl tritt bei tiefen Temperaturen (Abb. 6 unten) eine kräftige Verbreiterung der Linie ein, die sich im schnelleren Abfall des freien Präzessionssignals ausprägt.

Für die $T_{2 \mathrm{e}}$-Bestimmung, vor allem aber, um Auskunft über eine mögliche Temperaturabhängigkeit des Elektronenaustausches zu erhalten, wurden die Elektronenresonanzlinien gemessen. In Abb. 7 ist die Temperaturabhängigkeit des Abstandes der Extrema in den registrierten Ableitungskurven dargestellt.

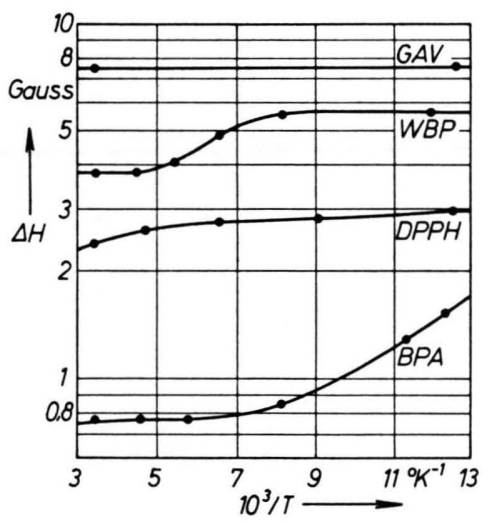

Abb. 7. Linienbreiten (Abstände der Extrema in den registrierten Ableitungskurven) der Elektronenresonanz in Gauss in Abhängigkeit von der Temperatur. Die Linienformen besitzen weitgehend Lorentz-Charakter.

\section{Diskussion}

Bei allen vier untersuchten Festkörperradikalen wurde eine positive dynamische Protonenpolarisation beobachtet. Die Ergebnisse der Abb. 3 und 4 entsprechen einem Overhauser-Effekt, der bei Sättigung der Elektronenresonanz auftritt, wenn zwischen Protonen und ungepaarten Elektronen eine zeitabhängige skalare Kopplung von $\operatorname{der} \operatorname{Art} A \mathbf{S}$ I besteht. Die Zeitabhängigkeit kann nur durch die begrenzte Lebensdauer $\tau$ der Elektronenspins in einem gegebenen Zustand zustandekommen. Die für die „flip-flop“-Prozesse des skalaren Overhauser-Effekts benötigte Energie muß also dem Spektrum des 
Schwankungsoperators $\mathbf{S}(t)$ entnommen werden. Mögliche Mechanismen für die statistischen Schwankungen von $S$ sind Austauschkopplungen $J S_{1} S_{2}$ zwischen benachbarten Elektronenspins und die Elektronenspinrelaxation.

Austauschwechselwirkungen sind aus der Theorie des Elektronenmagnetismus gut bekannt. Einen Hinweis für die Stärke der Austauschkräfte in den hier studierten freien Radikalen gibt Abb. 7. Während die gleichen Radikale in Lösung Hyperfeinstrukturspektren besitzen, die sich über einen breiten Magnetfeldbereich erstrecken, treten im kristallinen Zustand verengte Linien auf, deren Breite teilweise von der Temperatur abhängt. Die Verengung ist am stärksten bei BPA, die Lebensdauer $\tau$ eines Elektronenspinzustandes bei Zimmertemperatur ist hier von der Größenordnung $10^{-9} \mathrm{~s}$, für WBP $10^{-8} \mathrm{~s}$ oder kürzer. $\tau$ läßt sich abschätzen, indem man als Bedingung für die Ausmittelung eines bestimmten Linienbreitenbeitrages $\gamma_{S} \Delta H_{\mathrm{f}}$ ansetzt:

$$
1 / \tau \approx\left(\gamma_{S} \Delta H_{\mathrm{f}}\right)^{2} /\left(\gamma_{S} \Delta H\right)
$$

mit $\tau \gamma_{S} \Delta H_{\mathrm{f}} \ll 1$. Im Falle von WBP wird gerade eine Dispersionsstufe im untersuchten Temperaturbereich erfaßt, für die dann $\tau \gamma_{S} \Delta H_{\mathrm{f}} \approx 1$ gilt. Bei GAV erfolgt der Austausch so langsam, daß die durch Hyperfeinwechselwirkung mit dem Brückenproton entstehende Struktur noch nicht ausgemittelt ist.

Diese Aussagen werden durch die Form der Protonenresonanzlinien bestätigt. Die Linienbreiten entsprechen größenordnungsmäßig denen, die man bei alleinigem Vorhandensein von Dipolwechselwirkungen zwischen den Protonen im starren Gitter erwarten würde. Im Falle des GAV erfolgt der freie Induktionsabfall bei höheren Temperaturen langsamer als bei tieferen Temperaturen, Abb. 6, was auf $\mathrm{CH}_{3}$-Gruppenrotationen ${ }^{13}$ zurückzuführen ist. Die Dispersionsstufe für diesen Bewegungsprozeß liegt bei etwa $-100{ }^{\circ} \mathrm{C} \quad\left(1 / \tau_{\mathrm{r}} \approx \gamma_{I} \Delta H_{\mathrm{Kern}, \text { starr }}\right)$. Die starken, von den Elektronen herrührenden lokalen Magnetfelder von der Größenordnung $1 / \gamma_{I} \cdot A / \hbar$ wirken sich nicht oder nur unwesentlich auf die Linienbreite aus. Das bedeutet, daß für deren Schwankungsrate $1 / \tau \gg A / \hbar$ gelten muß. Bei einer Kopplungskonstanten in der Größenordnung $A / \hbar=10 \mathrm{MHz}$ heißt das, $\tau \ll 10^{-8} \mathrm{~s}$. Lediglich für GAV bei tiefen Temperaturen (Abb. 6 unten) wird $\tau$ unter Um-

13 J. G. Powles u. H. S. Gutowsky, J. Chem. Phys. 2l, 1704 [1953]. ständen so lang, daß zumindest die stärker gekoppelten Brückenprotonen ein nur unvollständig ausgemitteltes lokales Feld der Elektronenspins "sehen“. Eine quantitative Behandlung wird dadurch erschwert, daß die Elektronenspinrelaxationszeiten hier nicht mehr wesentlich länger sind (Tab. 1), und deren Temperaturabhängigkeit noch nicht bekannt ist.

Die Austauschraten oder die elektronische Relaxation sind in allen Fällen schnell genug, um einen Overhauser-Effekt zu erklären. Die Relaxationsstärke der Protonen bei skalarer Wechselwirkung ist allerdings proportional $\left[1+\omega_{S}^{2} \tau^{2}\right]^{-1}$, aber $\omega_{S}^{2} \tau^{2}$ ist nicht so viel größer als 1 , als daß das Schwankungsspektrum nicht die erforderlichen Energiequanten $\hbar \omega_{S}$ bereitstellen könnte. Im Gegensatz zu zeitabhängigen Dipolkopplungen hängt die Stärke der dynamischen Kernpolarisation bei reiner Skalarkopplung zunächst nicht von $\omega \tau$ ab, für den Kopplungsparameter $\varrho$ in (1) gilt $\varrho=-1$. In BPA wird der nach (1) mit $\varrho=-1$ und $f=1$ erwartete Vergrößerungsfaktor $P / P=+658$ nicht ganz erreicht. Da ein zweiter, von der Kopplung mit den Elektronen unabhängiger Relaxationsmechanismus $(f<1)$ in diesen Stoffen kaum wahrnehmbar ist, kann dafür ein geringer Einfluß von Dipolwechselwirkungen verantwortlich sein. Das ist insofern nicht ausgeschlossen, als der Dipol-Wechselwirkungsoperator Terme enthält, bei denen das Umklappen eines Protonenspins nicht mit dem gleichzeitigen Umklappen eines Elektronenspins verbunden sind. Für solche Prozesse braucht das Schwankungsspektrum nur die viel kleineren Energiequanten $\hbar \omega_{I}$ bereitzustellen, so daß auch bei schwacher Dipolkopplung oder niedriger Schwankungsrate des zeitabhängigen Operators derartige Einflüsse möglich sind. Die experimentellen Ergebnisse bei allen Radikalen zeigen indessen, daß der skalare Wechselwirkungsmechanismus sehr stark vorherrscht. Für weitergehende Aussagen müßte die Frequenzabhängigkeit der dynamischen Kernpolarisation bekannt sein.

Auch die Protonenrelaxationszeiten können in ihrer Größenordnung durch die skalaren Wechselwirkungen erklärt werden. Man kann ähnlich wie bei Flüssigkeiten argumentieren und die gebräuchliche Beziehung ${ }^{14}\left(\omega_{S} \gg \omega_{I}\right)$

$$
\frac{1}{T_{1}}=\frac{1}{2}(A / \hbar)^{2} \frac{\tau}{1+\omega_{S}^{2} \tau^{2}}
$$

14 A. Abragam, The Principles of Nuclear Magnetism, Clarendon Press, Oxford 1961, Kap. VIII. 
benutzen. Nach dem Vorigen ist $\omega_{S} \tau \gg 1$, und die Abnahme der Relaxationsstärke mit fallender Temperatur wird wiedergegeben. Allerdings hätte man bei BPA und DPPH einen etwas stärkeren Temperaturgang erwartet. Der steile Abfall bei WBP kann mit der Anomalie der Elektronenspinresonanz in Zusammenhang gebracht werden. Die Intensität der ESR-Signale nimmt bei tiefen Temperaturen kräftig ab, was nur durch ein Verschwinden des Radikals erklärt werden kann. Es gibt in der Literatur Hinweise ${ }^{15}$, daß sich die Spins in WBP bei tiefen Temperaturen paaren und die verbleibende Resonanz einem schwach angeregten Triplettzustand zuzuordnen ist. Die kleineren $1 / T_{1}$-Werte für GAV sind durch die schwachen Austauschraten verständlich. Der unübersichtliche Temperaturgang dürfte damit zusammenhängen, daß Austausch und Elektronenspinrelaxation gleichzeitig Einfluß besitzen. In allen beobachteten Fällen, auch bei verschieden starker Kern-Elektronen-Kopplung für nichtäquivalente Protonen ließ sich der Abfall der $H_{0}$-Komponente der Protonenmagnetisierung recht gut durch eine Exponentialfunktion annähern.
Wenn auch die vorliegende Arbeit noch einige Fragen offenließ, so ist doch sichergestellt, daß man bei den untersuchten organischen freien Radikalen dynamische Kernpolarisationen erzeugen kann, die dem theoretischen Wert sehr nahe kommen. Der Overhauser-Effekt kann nur durch eine vorherrschend skalare zeitabhängige Kopplung zwischen den Radikalelektronen und den Protonen in den Molekülen erklärt werden. Die Austauschverschmälerung der ESR-Spektren, die Form der Protonenresonanzlinien und die Spin-Gitter-Relaxation der Protonen stehen in Einklang mit der Annahme, daß die Lebensdauerbegrenzung der Elektronenspinzustände durch Elektronenaustausch den wirksamen Schwankungsmechanismus darstellt. Zwecks weiterer Informationen müßten die Frequenz- und Temperaturabhängigkeit der Kernpolarisation, die Relaxationsmechanismen der Elektronen und die Abhängigkeit des Austausches von den chemischen Herstellungsmethoden der Radikale genauer untersucht werden.

Der Deutschen Forschungsgemeinschaft, Bad Godesberg, danken wir für die Bereitstellung einer Beihilfe zur Unterstützung dieser Arbeit im Rahmen des Schwerpunktprogramms Hochfrequenzphysik.

15 McConnell, D. Poaley u. A. Bradbury, Proc. Nat. Acad. Sci. US 48, 1480 [1962]. 